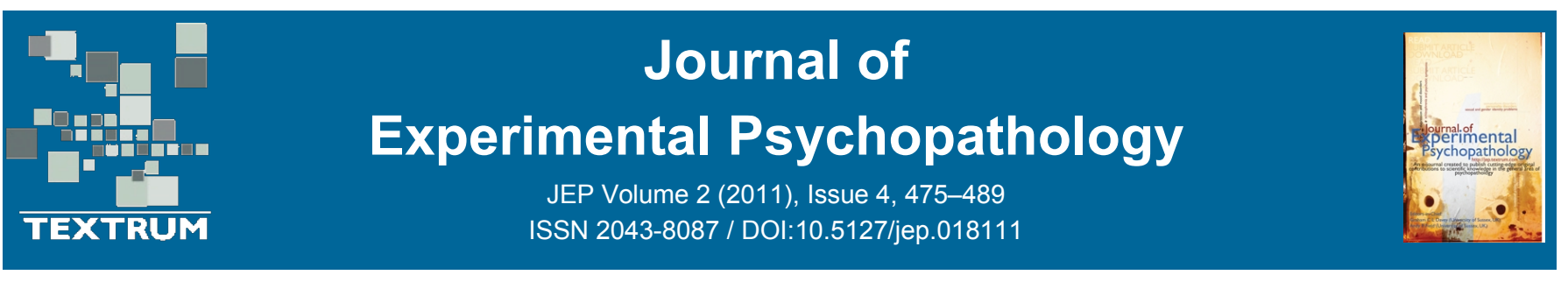

\title{
Attention Bias for Angry Faces in Children with Social Phobia
}

\author{
Allison M. Waters ${ }^{a}$, Karin Mogg ${ }^{b}$, Brendan P. Bradley ${ }^{b}, \&$ Daniel S. Pine \\ ${ }^{a}$ Griffith University, Australia \\ ${ }^{b}$ University of Southampton, UK \\ ${ }^{c}$ National Institute of Mental Health, USA
}

\section{Abstract}

Attention bias towards threatening stimuli is a well-established cognitive correlate of anxiety disorders. In tasks using angry faces, accumulating results link paediatric anxiety to biased attention. Nevertheless, at least in childhood social phobia, there is mixed evidence regarding the direction of this bias as some findings suggest that socially anxious children show increased attention bias towards threat, whereas other evidence suggests they have an increased bias away from threat. The present study examined attention bias for angry (and happy) faces in 27 children with a principal diagnosis of social phobia and 27 non-clinical healthy volunteers between 5 and 13 years of age. Children completed a visual probe task in which pairs of angry and neutral faces and happy and neutral faces were presented for $500 \mathrm{~ms}$ followed by an asterisk probe in either the same or opposite location as the emotional face. Participants were instructed to respond quickly by key-press to indicate the position of the probe. Results showed that only socially phobic children with high symptom severity showed a significant attention bias towards angry faces relative to neutral ones. In contrast, children with social phobia with lower levels of symptom severity showed a significant attention bias away from angry faces. Non-anxious volunteers showed no significant bias for angry faces. There were no significant between-group differences for happy faces. Results are discussed in terms of prior work on attention and emotion regulation.

(c) Copyright 2011 Textrum Ltd. All rights reserved.

Keywords: attention bias; angry faces; children; social phobia

Correspondence to: Dr Allison Waters, School of Psychology, Griffith University, Australia, Phone: 61-7-3735 3434; email: a.waters@griffith.edu.au

1. School of Applied Psychology, Griffith University, Mt Gravatt campus, Q 4122, Australia

2. School of Psychology, Shackleton Building, Univeristy of Southampton, Highfield campus, Southampton SO17 1BJ, UK.

3. National Institute of Mental Health, Bethesda MD 20892, USA

Received 09-Nov-2010; received in revised form 22-Apr-2011; accepted 27-Apr-2011 


\title{
Table of Contents
}

\author{
Introduction \\ Method \\ Participants \\ Measures \\ Diagnostic status. \\ Symptom measures. \\ Attention bias. \\ Procedure \\ Anxious children. \\ Healthy Volunteer Children. \\ Data Analysis \\ Results \\ Group characteristics \\ Visual probe task \\ Angry faces. \\ Happy faces. \\ Correlations between anxiety measures and attention bias scores \\ Discussion \\ Limitations \\ Clinical Implications \\ References
}

\section{Introduction}

Social phobia is a debilitating psychological disorder characterized by a fear of one or more social or performance situations. Although once viewed as a relatively-benign form of simple shyness, social phobia is now recognized to cause significant impairment in children and adolescents (LaGreca \& Lopez, 1998; Spence, Donovan, \& Brechman-Toussaint, 1999). Moreover, in youth, social phobia is relatively common with between $1 \%$ and $9 \%$ of community youth and up to $32 \%$ of clinically referred children meeting criteria for social phobia (Kendall et al., 1997; Last, Perrin, Hersen, \& Kazdin, 1992; Wittchen, Stein, \& Kessler, 1999). Furthermore, if left untreated, childhood social phobia can lead to other psychological disorders including substance use and depression (Kendall, Safford, Flannery-Schroeder, \& Webb, 2004; Kessler et al., 1996). Thus, the costly and debilitating nature of the condition highlights the need for further research examining mechanisms that contribute to the development and/or maintenance of childhood social phobia.

General cognitive models of anxiety (e.g., Mogg \& Bradley, 1998; Williams, Watts, MacLeod, \& Mathews, 1997) emphasize the role of an attention bias to threat in the causation and/or maintenance of anxiety, whereby anxious individuals selectively direct cognitive resources towards threat stimuli, especially during the very early stages of stimulus processing. However, specific cognitive models of social phobia propose competing hypotheses with regard to whether attention is directed towards or away from threat in social phobia. For example, consistent with general cognitive models, Rapee and Heimberg (1997) proposed that socially anxious individuals show enhanced selective attention towards threat cues, such as human faces signalling disapproval or criticism. By contrast, Clark and Wells (1995) proposed that socially anxious individuals direct attention away from external threat cues and towards internal cues of threat (i.e., self-focused attention), which, in turn, maintains the anxious state (Clark, 1999). Thus, on the one hand, individuals with social phobia should show increased selective attention 
towards threat stimuli, such as angry faces, while on the other hand, they are expected to show avoidance of external cues depicting social threat (Clark, 1999).

Consistent with these competing positions, data also appear inconsistent concerning the nature of attention bias in social phobia. The most current work in this area relies on the visual-probe task with emotional words or pictures to examine attention biases for threat. In this task, paired stimuli (e.g., threat-neutral word pair; angry-neutral face pair) are presented simultaneously on a computer screen for specified durations of time. The stimuli then disappear and are followed by a visual-probe (e.g. small dot) presented in the spatial location of one of the stimuli to which participants respond. Faster responsetimes (RTs) to probes replacing threat compared with neutral stimuli reflect an attention bias toward threat.

In adults, studies of social phobia generate mixed results regarding the direction of the threat attention bias. For example, Mogg and Bradley (2002) found enhanced vigilance for briefly presented (17 ms) angry faces, relative to neutral faces, in nonclinical social anxiety using a visual probe task. Similarly, Mogg, Philippot, and Bradley (2004) found that adults with clinical social phobia displayed an initial attention bias towards angry relative to neutral faces that dissipated over time: i.e., they showed initial bias towards angry faces at $500 \mathrm{~ms}$, but did not differ from non-anxious adults at $1250 \mathrm{~ms}$. In contrast, Mansell, Clark, Ehlers, and Chen (1999) found that nonclinical socially anxious adults avoided emotional faces (both positive and negative faces, relative to household objects) presented for $500 \mathrm{~ms}$ in a visual probe task, when under social-evaluative stress. Similarly, Chen, Ehlers, Clark, and Mansell (2002) found greater avoidance of negative, positive, and neutral faces (relative to household objects) when using the same task in adults with social phobia compared with non-anxious controls. Mogg et al. (2004) discussed these differences in terms of differing methodology whereby the use of inanimate household objects rather than faces as the control stimuli in the studies of Mansell et al. (1999) and Chen et al. (2002) may have given the social phobic adults an option for avoiding threatening faces.

While research in children has lagged behind work with adults, there is a particularly pressing need for work on childhood social phobia. There is accumulating evidence of a threat attention bias in children with anxiety disorders, including generalised anxiety disorder (GAD) (Taghavi, Dalgleish, Moradi, Neshat-Doost \& Yule, 2003; Waters, Mogg, Bradley, \& Pine, 2008; Monk et al., 2006; 2008), posttraumatic stress disorder (PTSD) (Dalgliesh et al., 2003; Pine et al., 2005), separation anxiety disorder (In-Albon, Kossowsky, \& Schneider, 2010), and high trait anxiety (Telzer et al., 2008; Waters, Kokkoris, Mogg, Bradley, \& Pine, 2010; Watts \& Weems, 2006). One relatively large study examined attention biases among children with GAD, separation anxiety disorder, and social phobia (Roy et al., 2008). However, beyond such studies of mixed paediatric anxiety disorders (Roy et al., 2008; Waters, Henry et al., 2010) or other work on non-clinical forms of social anxiety (Stirling, Eley, \& Clark, 2006; Perez-Edgar et al., 2010), no study has examined attention bias in children selected specifically for the diagnosis of social phobia.

The few available directly relevant studies find mixed results, which could reflect differences in methodology or the characteristics of children that have been studied. In clinical studies, Waters, Henry et al. (2010) found that an attention bias towards angry faces presented for $500 \mathrm{~ms}$ in a visual probe task was characteristic of clinically anxious children between 8 and 11 years of age, including those with social phobia. However, the sample size was too small to examine specific disorder effects. A larger study utilising the same task and exposure duration targeted children who specifically suffered from relatively severe anxiety that did not respond to an initial brief period of psychotherapy (i.e., Roy et al., 2008). In this study, Roy et al. (2008) found that attention bias towards angry faces was common to all clinically anxious children and adolescents (7-17 years of age) relative to controls. Moreover, there were 
no differences found in the attention bias towards angry faces among children with GAD, separation anxiety disorder and social phobia.

Finally, in a non-clinical study of school children between 8 and 11 years of age, Stirling et al. (2006) found a positive association between attention bias away from negative faces (particularly angry and fearful faces) and increased social anxiety symptoms. It is noteworthy that aspects of Stirling et al.'s (2006) study differed from the clinical studies, in that they employed a $1000 \mathrm{~ms}$ exposure duration which may have specifically tapped into later avoidance stages of information processing. Another difference is that attention bias towards angry faces was observed in children with severe levels of anxiety symptoms in Waters, Henry et al. (2010) and Roy et al. (2008) whereas a non-clinical school-age sample was studied in Stirling et al. (2006). Thus, the nature of attention bias in anxious children may vary with subtle task-related features or with the severity of a child's anxiety; moderately anxious children may direct attention away from angry faces, potentially as one means to regulate negative emotion, whereas more severely anxious children fail at this, and thus, attend disproportionately to threat.

Thus, the preliminary evidence on attention bias in paediatric social phobia generates conflicting findings; the direction of attention bias appears to vary as a function of symptom severity (Roy et al., 2008; Stirling et al., 2006). To add to this preliminary evidence, the aim of this study is to examine attention bias for angry faces in children with a principal diagnosis of social phobia, compared with healthy peers, and whether this bias varies as a function of symptom severity. Specifically, we were interested in whether an attention bias to angry faces would be more apparent in children with higher levels of anxiety symptoms (cf. Roy et al., 2008; Waters, Henry et al., 2010), and whether children with lower levels of symptoms would show a reduced bias, or possibly even a bias away from angry faces (cf. Stirling et al., 2006). This study also addressed design and methodological variability across prior studies by employing a well-validated visual probe task with a $500 \mathrm{~ms}$ exposure duration to assess early attention processes that has been used widely in prior child and adult studies (e.g., Mogg et al., 2004; Pine et al., 2005; Roy et al., 2008; Waters, Mogg et al., 2008; Waters, Henry et al., 2010; Waters, Kokkoris et al., 2010). The angry face stimuli were paired with neutral faces as control stimuli, which is consistent with most previous research. The task also included pairs of happy and neutral faces, which has the advantage that not all the emotional stimuli were negative, which could be unsettling for young children. The inclusion of these stimuli also allows assessment of attention bias for happy faces. However, the majority of studies show no relationship between anxiety and happy face attention bias in youth (e.g. Roy et al., 2008; Stirling et al., 2006; Waters, Kokkoris et al., 2010) with only one study suggesting a positive association (Waters et al., 2008), so we did not expect to find group differences in this bias.

\section{Method}

\section{Participants}

Participants included 54 children aged between 5 years, 0 months and 13 years, 2 months ( 23 males, 31 females): 27 anxious children (10 males, 17 females) and 27 non-anxious healthy volunteer children (13 males, 14 females). No data on attention bias in these children have been previously published. The social phobia group was further divided based on anxiety severity scores on the Spence Children's Anxiety Scale (Nauta et al., 2004; Spence, 1998) to form a social phobia group with low anxiety severity (SP-LAS, $N=14$ ) and a social phobia group with high anxiety severity (SP-HAS, $N=13$ ) (see Measures for further details). 
Anxious children were recruited via advertisements distributed to local community agencies and primary schools. Non-anxious control children were recruited from a local primary school. Ninety-three percent of children were born in Australia, 2\% in New Zealand, 2\% in South Africa and 3\% described as "other". All of the children spoke English as their first language. Eighty-five percent of children lived with parents who were married, $5 \%$ had parents who were divorced, and $10 \%$ had parents who were either separated, living in de facto arrangements, or were never married. Seventy-eight percent of mothers and $97 \%$ of fathers were employed full time. Using the Daniel Prestige Scale (Daniel, 1983), which provides a rating of Australian occupational prestige $(0=$ high to $7=\mathrm{low})$, mean scores of $4.91(S D=1.23)$ and $4.04(S D=0.88$ ) for mothers' and fathers' occupations, respectively, indicate the majority of children came from average-income Australian families. There were no significant group differences on any of these demographic variables.

\section{Measures}

\section{Diagnostic status.}

Diagnostic interviews were performed using the parent interview schedule of the Anxiety Disorders Interview Schedule for the fourth edition of the Diagnostic and Statistical Manual of Mental Disorders (DSM-IV): Child / Parent Versions (ADIS-C-IV-C/P; Silverman \& Albano, 1996). Only children with an ADIS-C-IV-C/P clinical severity rating (CSR) of four or higher for their principal anxiety diagnosis (i.e., most severe) were included. The telephone administration of the ADIS-C-IV-C/P was used for determining diagnosis and possesses good inter-rater reliability (Cobham, Dadds, \& Spence, 1998), a high level of agreement with face-to-face administration (Lyneham \& Rapee, 2005), and sound psychometric properties (Rapee, Barrett, Dadds, \& Evans, 1994; Silverman, Saavedra, \& Pina, 2001).

\section{Symptom measures.}

The parent- and child-report formats of the Spence Children's Anxiety Scale (SCAS-P, SCAS-C) (Spence, 1998) were utilised to assess anxiety symptoms. The SCAS-P, a 39 item parent report measure, and the SCAS-C, a 45-item child self-report measure (6 positive filler items), both contain 4point response scales ( $0=$ never true to $3=$ always true) and yield total and subscale scores in accordance with the DSM-IV anxiety diagnoses and possess sound psychometric properties (Nauta et al., 2004; Spence, 1998). Only children from 8 years and older $(N=29)$ completed the SCAS-C, consistent with the normative data, and to prevent fatigue and loss of concentration in younger children. Mean SCAS-P total scores of 14.20 and 31.80 and mean SCAS-C total scores of 18.80 and 32.20 have been reported for non-clinical controls and clinically-anxious children respectively (Nauta et al., 2004; Spence, 1998).

Anxiety severity within the social phobia group was examined by forming two distinct groups based on children scoring above and children scoring below the median SCAS-P social phobia subscale score for the group as a whole. The SCAS-P rather than the SCAS-C was used since children younger than 8 years did not have SCAS-C data. A median split on the SCAS-P social phobia score produced the same group allocation as that produced by a median split on the SCAS-P total score, given the high comorbidity between anxiety diagnoses; so it is consistent with our prior study for comparison purposes to use the SCAS-P total score to form severity groups (Waters et al., 2008). Thus, the mean SCAS-P total score for the social phobia group with low anxiety severity (SP-LAS; $N=14$ ) and high anxiety severity (SP-HAS; $N=13$ ) (see Table 1) are comparable to the means observed for low and high anxiety severity groups in our prior studies (e.g., Waters et al., 2008; low = 23.40; high; 42.38; Waters, Henry et al., 2010; low = 21.85; high $=46.38$ ). 
Table 1: Means and standard deviations (in parentheses) for characteristics of social phobia and control groups (SP-LAS = social phobia-low anxiety severity; SP-HAS = social phobia-high anxiety severity)

\begin{tabular}{lccc}
\hline Characteristic & Controls & SP-LAS & SP-HAS \\
\hline Age (Years, Months) & $8.40(1.7)$ & $8.65(2.05)$ & $8.60(1.90)$ \\
Gender (\%Male) & $37 \%$ & $42 \%$ & $30 \%$ \\
SCAS-P Total & $11.42(6.04)$ & $19.19(7.99)$ & $39.37(7.95)$ \\
SCAS-P Social & $1.36(1.25)$ & $6.70 \mathrm{~b}(2.83)$ & $11.53(3.12)$ \\
SCAS-C Total & $16.50(7.78)$ & $38.00(12.93)$ & $48.00(24.96)$ \\
SCAS-C Social & $2.24(1.53)$ & $6.18(3.70)$ & $10.23(4.81)$ \\
Mean Number of Diagnoses & - & $2.00(1.79)$ & $2.61(1.38)$ \\
\hline
\end{tabular}

\section{Attention bias.}

The visual probe task was programmed using E-prime v1.1 (Psychology Software Tools, Inc) and presented on two identical Dell Optiplex computers with a 17" $75 \mathrm{~Hz}$ CRT colour monitor: one at the primary schools and one in a research laboratory at Griffith University. The main stimuli were grey-scale photographs of face-pairs from 64 different actors (half male, half female), each presenting a neutral and either an angry or happy expression (see Bradley, Mogg, Falla \& Hamilton, 1998, for detailed description of the face stimuli). Both these faces and this task design are the same as used in many prior studies, including six prior studies in children, as reported by Pine et al. (2005), Monk et al. (2006), Roy et al., (2008), and Waters, Mogg et al., (2008); Waters, Henry, Mogg, Bradley \& Pine, (2010); Waters, Kokkoris et al., (2010), as well as in several studies of adult anxiety (e.g. Bradley et al., 1998, 1999; Mogg \& Bradley, 1999; Mogg et al., 2004). There were 32 angry-neutral and 32 happy-neutral face-pairs, and 16 neutral-neutral face-pairs which were used on filler trials. The facial expressions shown in each face pair were from the same individual and different individuals were used for angry-neutral, happy-neutral and neutral-neutral face pairs. The stimuli used in the practice trials were selected from an additional 10 face pairs.

\section{Procedure}

\section{Anxious children.}

After initial referral and phone screening with parents, children and parents in the anxious group attended an assessment session at Griffith University. Families were given the information sheet, family demographic form and consent form pertaining to this study at the start of the session. The ADIS-C-IV$\mathrm{C} / \mathrm{P}$ was then administered with the attending parent/s by a clinical psychology doctorate student trained in the ADIS-C-IV-C/P. Children completed the visual probe task followed by the SCAS-C with a research assistant in a research laboratory. Parents completed the SCAS-P during this time. All anxious children completed the assessment session prior to cognitive-behavioural treatment for anxiety disorders.

\section{Healthy Volunteer Children.}

School children in grades Prep to 7 (i.e., 4.5 to $12-13$ year olds) at two local primary schools were recruited for this study. Education department and school approvals were obtained before commencing the study. Children were sent home with the same information sheet, consent form, family demographic form, and the SCAS-P as used with the anxious group. Children whose parents gave consent returned the completed forms to a box in their classroom. Parents of consented children with the lowest SCAS-P scores were telephoned by a senior clinical graduate student within one month after initial consent was given and the ADIS-C-IV-C/P was administered over the phone with available parents (primarily 
mothers). Healthy volunteer children then completed the visual probe task and questionnaires individually in a resource room at the primary school. This was done within two weeks of the phone call to parents to complete the ADIS-C-IV-C/P. A research assistant supervised children at all times and the session lasted approximately $45 \mathrm{~min}$.

The procedure for the visual probe task was similar to that used in previous studies (e.g., Mogg \& Bradley, 1999; Roy et al., 2008; Waters, Mogg et al., 2008). Each trial began with a 500 ms fixation point followed by two simultaneously presented faces on the left and right of the screen for $500 \mathrm{~ms}$. The facepair was replaced with an asterisk (probe) which was centred in the spatial location previously occupied by one of the faces and always appeared at the same location on either the left or right side of the screen. Participants were instructed to press one of two keys labelled "left" and "right" as quickly as possible while avoiding mistakes, to indicate whether the asterisk appeared on the left or right. Thus, a probe-position (rather than probe-classification or probe-detection) format was employed as this format is simple and commonly used with children in prior studies (e.g., Pine et al., 2005; Monk et al., 2006; Roy et al., 2008; Waters, Mogg et al., 2008, Waters, Henry et al., 2010; Waters, Kokkoris et al., 2010). The inter-trial interval varied randomly from 750 to $1250 \mathrm{~ms}$. The task began with 10 random practice trials, followed by one block of 80 trials. On critical trials (i.e. angry-neutral or happy-neutral face-pairs), the emotional face and the asterisk appeared on either the left or right side on the screen with equal frequency, so that on half the trials the probe appeared in the same spatial location as the emotional face (congruent trials), and on the other half of trials the probe appeared in the opposite location to the emotional face (incongruent trials). The order of trials was randomly determined for each participant.

\section{Data Analysis}

Analyses were based on mean reaction times (RTs) to probes in ms. RTs from trials with incorrect responses were excluded. Outliers were identified as RTs less than $200 \mathrm{~ms}$ or more than $3 \mathrm{SDs}$ above each participant's mean. There was no significant difference between the groups in the amount of data lost due to either errors (less than 1\% of trials) or outliers (less than 1\%) and there were no significant differences between groups in mean RT (control $=571.66(S D=88.16)$; SP-LAS $=643.44(S D=$ 121.70); SP-HAS = $594.99(S D=127.52)$.

Analyses were conducted on attention bias scores which were derived from the RT data. RTs to probes were averaged separately for congruent and incongruent trials in each location. Bias scores were calculated separately for angry and happy faces by subtracting the average RT on congruent trials from the average RT on incongruent trials. Positive values of bias scores indicate attention toward the angry (or happy) face relative to neutral faces, whereas negative values indicate attention away from the angry (or happy) face.

Analyses were conducted separately for angry and happy face bias scores, given the absence of specific theory-driven predictions regarding happy faces. The initial analysis of attention bias scores for angry faces, relative to neutral faces, was conducted using analysis of variance (ANOVA) with two levels of Group [SP; control]. We subsequently investigated the effect of anxiety symptom severity on attention bias in an ANOVA with three levels of Group [SP-LAS; SP-HAS; control]. Similar analyses were used to examine bias scores for happy relative to neutral faces. Follow-up tests of significant effects were based on t-tests with Bonferroni adjustments for multiple comparisons. Partial eta squared $\left(\eta_{p}{ }^{2}\right)$ was calculated as an estimate of effect size. 


\section{Results}

\section{Group characteristics}

The groups differed significantly on a range of diagnostic and symptom measures (see Table 1). Given that these groups were formed based on their symptom profiles, significant differences were expected between the groups on SCAS-P and SCAS-C total scores, $F(2,51)=65.60, p<.001, \eta_{p}{ }^{2}=.72$, and $F(2$, $26)=11.18, p<.001, \eta_{p}{ }^{2}=.46$, and on SCAS-P and SCAS-C social anxiety subscales scores, $F(2,51)$ $=58.02, p<.001, \eta_{p}{ }^{2}=.69$, and $F(2,26)=18.14, p<.001, \eta_{p}{ }^{2}=.49$. As expected, the SP-HAS group had significantly higher SCAS-P total scores and social anxiety subscale scores than the healthy and SP-LAS groups (all $p s=.011$ ). The SP-LAS group also had significantly higher SCAS-P total scores and social anxiety subscale scores than healthy volunteers (all $p s=.001$ ). On the SCAS-C, the SP-HAS group had significantly higher social anxiety subscale scores than the SP-LAS group $(p=.01)$ and healthy volunteers $(p=.001)$. Similarly, the SP-LAS group also had significantly higher social anxiety subscale scores than the volunteer group $(p=.007)$. However, SCAS-C total scores did not differ significantly between the SP-HAS and SP-LAS groups $(p=.62)$. Finally, there were no significant differences in the number of diagnoses between the SP-HAS and SP-LAS groups, $t(25)=0.99, p=.33$. There also were no significant age, $F(2,51)=0.12, p<.88, \eta_{p}{ }^{2}=.05$ or gender differences, $\chi(2)=1.01$, $p=.60$, between the SP-HAS, SP-LAS and control groups.

\section{Visual probe task}

\section{Angry faces.}

Figure 1 (upper panel) presents the mean attention bias scores for angry faces in each group (see Table 2 for mean RTs and bias scores). The ANOVA of bias scores which compared the combined SP group with controls revealed no significant effects, $F<1.99$, ns. However, the ANOVA of attention bias scores for angry faces, when examined as a function of anxiety severity, revealed a significant main effect of Severity Group, $F(2,51)=4.00, p=.024, \eta_{p}^{2}=.14$.

Table 2: Mean RTs on congruent and incongruent trials and attention bias scores (in ms) for angry and happy faces as a function of group (SP-LAS = social phobia-low anxiety severity; SP-HAS = social phobia-high anxiety severity)

\begin{tabular}{lcccccc}
\hline & \multicolumn{2}{c}{ Controls } & \multicolumn{2}{c}{ SP-LAS } & \multicolumn{2}{c}{ SP-HAS } \\
\cline { 2 - 7 } & $\boldsymbol{M}$ & $\boldsymbol{S D}$ & $\boldsymbol{M}$ & $\boldsymbol{S D}$ & $\boldsymbol{M}$ & $\boldsymbol{S D}$ \\
\hline Angry Congruent & 576.6 & 93.9 & 663.6 & 137.9 & 587.1 & 120.3 \\
Angry Incongruent & 577.5 & 94.2 & 642.1 & 129.9 & 605.9 & 147.1 \\
Angry Face Bias & 0.9 & 31.8 & -21.5 & 49.7 & 18.9 & 32.1 \\
Happy Congruent & 561.3 & 82.2 & 628.2 & 119.4 & 584.4 & 104.0 \\
Happy Incongruent & 568.1 & 92.5 & 636.9 & 124.8 & 593.5 & 110.1 \\
Happy Face Bias & 6.9 & 42.3 & 8.6 & 53.9 & 9.1 & 39.8 \\
\hline
\end{tabular}

Follow-up comparisons indicated that the SP-HAS group showed a significant attention bias towards angry faces, relative to neutral faces, as their mean attention bias score for angry faces was significantly greater than zero, $t(12)=2.12, p=.05$. By contrast, the SP-LAS group showed a significant attention bias away from angry faces, which also differed significantly from zero, $t(14)=2.31, p=.049$. Bias scores in control children did not significantly differ from zero, $t(26)=0.15, p=.88$. 
Group contrasts also revealed that attention towards angry faces in the SP-HAS group differed significantly from attention away from angry faces in the SP-LAS group $(p=.02)$. However, neither group differed significantly from controls (both $p s>.22$ ).

\section{Happy faces.}

Figure 1 (lower panel) presents the mean attention bias scores for happy faces in each group (see Table 2 for mean RTs). As can be seen, the groups did not differ in their attention towards happy faces. This was confirmed by a non-significant effect of Severity Group, $F(2,51)=0.15, p=.99, \eta_{p}{ }^{2}=.01$.
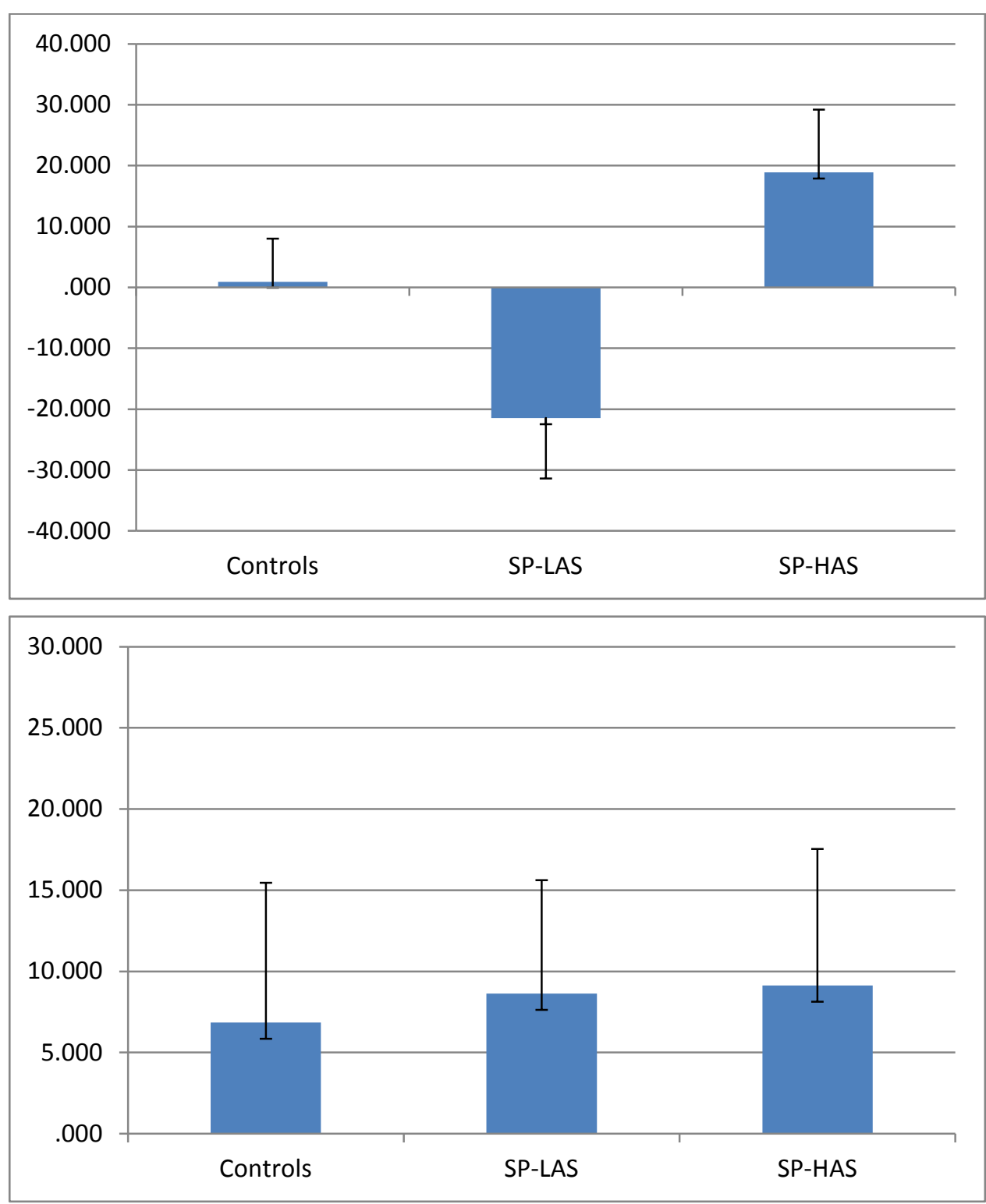

Figure 1: Mean attention bias scores (+SE) for angry (upper panel) and happy faces (lower panel) as a function of group (SP-LAS = social phobia-low anxiety severity; SP-HAS = social phobia-high anxiety severity)

\section{Correlations between anxiety measures and attention bias scores}

Correlations between anxiety measures and attention bias scores in the anxious group revealed a significant positive relationship between SCAS-P total scores and attention bias for angry faces $\left(r_{s}=.52\right.$, $p=.007)$. There also was a significant negative association between child total SCAS-C scores and attention bias for happy faces $\left(r_{s}=-.56, p=.018\right)$, reflecting that as child self-reported anxiety increased, children avoided happy faces. There was a significant positive association between angry 
face attention bias and comorbid diagnosis of GAD $\left(r_{p b}=.57 ; p=.016\right)$, but not separation anxiety disorder $\left(r_{p b}=.23 ; p=.24\right)$ or specific phobia $\left(r_{p b}=-.04 ; p=.84\right)$.

\section{Discussion}

The present study found that highly anxious children with a principal diagnosis of social phobia showed an attention bias towards angry faces. In contrast, children with social phobia exhibiting less severe symptoms directed their attention away from angry faces relative to neutral ones. Non-anxious children showed no attention bias for angry faces. As expected, there were no group differences in attention bias for happy faces.

The finding of an attention bias towards angry faces in the SP-HAS group is consistent with predictions from some cognitive theories of anxiety and social phobia (e.g., Mogg \& Bradley, 1998; Williams et al., 1997; Rapee \& Heimberg, 1997). This result also accords with prior adult social phobia studies (e.g., Mogg \& Bradley, 2002; Mogg et al., 2004) and childhood anxiety studies which found an attention bias towards angry faces was characteristic of anxious children with more severe levels of anxiety, including those with social phobia (Roy et al., 2008; Waters, Henry et al., 2010). Moreover, the fact that the correlation analyses showed that, within the social phobia group, the attention bias for threat was positively associated with increased anxiety severity and the presence of GAD is in line with our prior finding that attention towards angry faces was associated with a diagnosis of social phobia in children with a principal diagnosis of GAD (Waters, Mogg et al., 2008). Similar links between GAD, social phobia and attention towards angry faces have been observed in adult studies (Bradley, Mogg, White, Groom, \& de Bono, 1999). It should be noted that the high level of comorbidity between symptoms of general anxiety and social anxiety prevents strong conclusions regarding the specific relationship between the attentional bias and each type of symptom. Thus, the attention bias observed in the SP-HAS group may not be unique to social phobia, but may be more characteristic of a high level of clinical anxiety symptoms in general.

Despite this caveat, the present study and its findings are informative in several notable respects. First, to our knowledge, this is the first study to examine attention bias for angry faces in children who have been specifically selected on the basis of having a principal diagnosis of social phobia. Second, despite an apparent absence of an attentional bias in the social phobia group as a whole, the present results highlight the importance of examining the effect of anxiety symptom severity in children. Indeed, if this had not been examined, an erroneous conclusion may have been inferred; i.e. there was no detectable attention bias in children with clinical social phobia. These analyses were justified from previous research indicating that, in clinically anxious children, symptom severity is a critical variable determining whether or not an attentional bias is revealed (Waters, Mogg et al., 2008; Waters, Henry, et al., 2010). Given that this is now the third study to reveal a positive association between attentional bias and anxiety symptom-severity in children, the accumulating evidence for this association cannot easily be attributed to Type 1 error. Third, the results are important because they indicate that the attentional bias away from angry faces previously found in sub-clinical social anxiety (Stirling et al. 2006), and also in children with low-level clinical anxiety symptoms (in the present study), does not become more exaggerated as clinical anxiety symptoms increase. That is, the SP-HAS group not only had higher social and general anxiety symptoms than the SP-LAS group (evident from SCAS social and total anxiety scores), but they also showed a significantly greater attentional bias towards angry faces, rather than increased avoidance.

The finding of an attention bias away from angry faces in the SP-LAS group, who nevertheless had clinical levels of anxiety, contrasts with expectations arising from general cognitive theories of anxiety 
disorders or some theoretical accounts specific to social phobia specifically (Mogg \& Bradley, 1998; Rapee \& Heimberg, 1997; Williams et al., 1997). Although other theories predict avoidance of threat in social phobia (e.g., Clark \& Wells, 1995), they do not specify differences as a function of increasing symptom severity. Nevertheless, the bias away from angry faces in the SP-LAS group in the current study seems to resemble the bias observed by Stirling et al. (2006) in school-age children with high social anxiety scores. Although, as noted earlier, Stirling et al.'s (2006) findings could be attributed to tapping into later stages of information processing with the use of a $1000 \mathrm{~ms}$ exposure duration, this argument does not apply in the present study with a shorter (500 ms) exposure duration. Instead, findings from both Stirling et al.'s (2006) study and the present one suggest that an attention bias away from angry/negative faces is characteristic of children with social anxiety who have lower levels of symptom severity. This pattern of results indicates that the relationship between attention bias and anxiety symptoms should not be assumed to be a simple linear one across samples with non-clinical and clinical social anxiety. One possible explanation is that moderately anxious children might avoid angry faces as an emotion regulation strategy to prevent the escalation of distress and anxiety, but that children with higher levels of anxiety may have difficulty in using such strategies. This explanation accords with the view that a threat attention bias may be due, at least in part, to poor attention control (i.e., ability to effortfully focus and shift attention) in highly anxious individuals (e.g., Lonigan \& Vasey, 2009). It would seem informative for future research to examine in more detail the relationships between attention bias and attention control and both sub-clinical and clinical anxiety in children.

A subsidiary feature of the present study concerned attention bias for happy faces. There was no between-group effect of happy faces on attention bias. However, correlation analyses suggested that within the social phobic group, children who self-reported higher levels of anxiety showed reduced attention bias to happy faces, relative to neutral faces. Similar findings have been recently observed in boys with anxiety and mixed anxiety-depression (i.e., Hankin, Gibb, Abela, \& Flory, 2010) and in adolescents with a history of high behavioural inhibition (Perez-Edgar et al., 2010). However, other studies have found no significant relationship between anxiety and bias for happy faces (Roy et al., 2008; Stirling et al., 2006; Waters, Henry et al., 2010) or suggested a tendency for a positive relationship (Bradley et al., 1999; Waters, Mogg et al., 2008). Despite some mixed results, a recent meta-analysis of anxiety studies suggested that low anxious individuals preferentially attend to positive relative to neutral stimuli, and this bias is commonly reduced or reversed in high anxious and dysphoric individuals (Frewen, Dozois, Joanisse \& Neufeld, 2008). It has been suggested that biases to happy stimuli may aid emotion regulation under stress (Frenkel, Lamy, Algom, \& Bar-Haim, 2008; Wadlinger \& Isaacowitz, 2008), and this positive bias may help protect children from negative emotion and poor outcomes (Perez-Edgar et al., 2010). Thus, the negative correlation between bias for happy faces and social anxiety, observed in the present study, might index the loss of a protective mechanism that underlies resilience to stress. However, this finding should be interpreted with caution, as this relationship was only found in correlation analyses with SCAS-C scores, which were not available for the whole sample, because these scores could only be obtained for children aged 8 years and older.

\section{Limitations}

The high comorbidity between social phobia and other anxiety disorders, especially GAD, highlights a limitation of this study in that the sample size was too small to study attention bias effects in social phobia with and without comorbid anxiety disorders. Given that Waters, Mogg et al., (2008) and Bradley et al. (1999) point to the close association between social anxiety and attention bias for emotional faces in GAD, a future study that more carefully controls for the effects of comorbidity is required. A second limitation was that assignment to the SP-HAS versus the SP-LAS group was done on the basis of parent 
report of anxiety severity, as self-reported SCAS-C scores were not available for young children. Although the groups did not differ significantly in SCAS-C total scores, which may have been due to the smaller sample given that only children 8 years and older completed the SCAS-C, it is reassuring that SP-HAS and SP-LAS groups differed significantly in SCAS-C social anxiety scores. Third, a measure of attention control, such as a version of the Attention Control Scale (Derryberry \& Reed, 2002) adapted for children, would help elucidate to what extent the direction of attention bias for emotional faces in more and less severely anxious children is related to cognitive control. Finally, given that prior research has shown elevated responding to neutral faces in children (Waters, Neumann, Henry, Craske, \& Ornitz, 2008), their subjective judgements of the threat value of faces should be assessed in future research.

\section{Clinical Implications}

The present findings add to the accumulating literature on cognitive biases in childhood anxiety disorders. The present findings in combination with other studies, suggest that the direction of the bias in clinically anxious children can vary and is influenced by symptom severity. That is, higher symptom severity is associated with attention towards angry faces, as predicted by various cognitive models of social and general anxiety. In contrast, threat avoidance appears characteristic of lower symptom severity, and may reflect emotion regulation efforts via cognitive control to offset negative affect and distress. It currently is unknown what effect attention avoidance of threat may have in the developmental progression of anxiety in less severely anxious children. Longitudinal research aimed at delineating the parameters of attention bias in childhood anxiety disorders over time is needed and will likely facilitate the development of more effective treatments. For example, attention bias modification training aims to reduce anxiety by targeting the underlying mechanism of biased attention towards threat stimuli and typically involves training anxious individuals to avoid threat (e.g., Hakamata et al., 2010). The application of these interventions may require modification if attention in anxious children is not as uniformly biased towards threat stimuli as seen in anxious adults.

\section{References}

Bradley, B.P., Mogg, K., Falla, S.J. \& Hamilton, L.R. (1998). Attentional bias for threatening facial expressions in anxiety: manipulation of stimulus duration. Cognition and Emotion, 12, 737-753. http://dx.doi.org/10.1080/026999398379411

Bradley, B. P., Mogg, K., White, J., Groom, C., \& de Bono, J. (1999). Attentional bias for emotional faces in generalized anxiety disorder. British Journal of Clinical Psychology, 38, 267-278. http://dx.doi.org/10.1348/014466599162845

Chen, Y. P., Ehlers, A., Clark, D. M., \& Mansell, W. (2002). Patients with generalized social phobia direct their attention away from faces. Behaviour Research \& Therapy, 40, 677-687. http://dx.doi.org/10.1016/S0005-7967(01)00086-9

Clark, D. M. (1999). Anxiety disorders: Why they persist and how to treat them. Behaviour Research and Therapy, 37 Suppl 1, S5-S27.

Clark, D. M., \& Wells, A. (1995). A cognitive model of social phobia. In M. R. Liebowitz (Ed.), Social phobia: Diagnosis, assessment, and treatment (pp. 69-93). New York, NY: Guilford Press.

Cobham, V. E., Dadds, M. R., \& Spence, S. H. (1998). The role of parental anxiety in the treatment of childhood anxiety. Journal of Consulting and Clinical Psychology, 66, 893-905.

http://dx.doi.org/10.1037/0022-006X.66.6.893 
Dalgleish, T., Taghavi, R., Neshat-Doost, H., Moradi, A., Canterbury, R., \& Yule, W. (2003). Patterns of processing bias for emotional information across clinical disorders: A comparison of attention, memory, and prospective cognition in children and adolescents with depression, generalized anxiety, and posttraumatic stress disorder. Journal of Clinical Child and Adolescent Psychology. 32, 10-21.

Daniel, A. E. (1983). Power, Privilege and Prestige. Melbourne, Australia: Longman Cheshire.

Derryberry, D., \& Reed, M. A. (2002). Anxiety-related attentional biases and their regulation by attentional control. Journal of Abnormal Psychology, 111, 225-236. http://dx.doi.org/10.1037/0021843X.111.2.225

Frenkel, T. I., Lamy, D., Algom, D., \& Bar-Haim, Y. (2008). Individual differences in perceptual sensitivity and response bias in anxiety: Evidence from emotional faces. Cognition and Emotion, 22, 1-13.

Frewen, P. A., Dozois, D. J. A., Joanisse, M. F., \& Neufeld, R.W. J. (2008). Selective attention to threat versus reward: Meta-analysis and neural-network modeling of the dot-probe task. Clinical Psychology Review, 28, 307-337. http://dx.doi.org/10.1016/j.cpr.2007.05.006

Hakamata, Y, Lissek, S., Bar-Haim, Y., Britton, J, C., Fox, N., Leibenluft, E.,... Pine, D. S. (2010). Attention bias modification treatment: A meta-analysis towards the establishment of novel treatment for anxiety, Biological Psychiatry, 68, 982-990. http://dx.doi.org/10.1016/i.biopsych.2010.07.021

Hankin, B. J., Gibb, B. E., Abela, J. R. Z., \& Flory, K. (2010). Selective attention to affective stimuli and clinical depression among youths: Role of anxiety and specificity of Emotion. Journal of Abnormal Psychology, 119, 491-501. http://dx.doi.org/10.1037/a0019609

In-Albon, T., Kossowsky, J., \& Schneider, S. (2010). Vigilance and avoidance of threat in the eye movements of children with separation anxiety disorder. Journal of Abnormal Child Psychology, 38, 225-235. http://dx.doi.org/10.1007/s10802-009-9359-4

Kendall, P. C., Flannery-Shroeder, E., Panichelli-Mendel, S., Southam-Gerow, M., Henin, A., \& Warman, M. J. (1997). Therapy for youths with anxiety disorders: a second randomized clinical trial. Journal of Consulting and Clinical Psychology, 65, 366-380. http://dx.doi.org/10.1037/0022-006X.65.3.366

Kendall, P. C., Safford, S., Flannery-Schroeder, E. and Webb, A. (2004). Child anxiety treatment: outcomes in adolescence and impact on substance use and depression at 7.4-year follow-up. Journal of Consulting and Clinical Psychology, 72, 276-287. http://dx.doi.org/10.1037/0022-006X.72.2.276

Kessler, R. C., Nelson, C., McGonagale, K., Edlund, M., Frank, R., \& Leaf, P. (1996). The epidemiology of co-occurring addictive and mental disorders: implications for prevention and service utilization. American Journal of Orthopsychiatry, 66, 17-31. http://dx.doi.org/10.1037/h0080151

LaGreca, A. M. \& Lopez, N. (1998). Social anxiety among adolescents: linkages with peer relations and friendships. Journal of Abnormal Child Psychology, 26, 83-94. http://dx.doi.org/10.1023/A:1022684520514

Last, C. L., Perrin, S., Hersen, M. and Kazdin, A. E. (1992). DSM-III-R anxiety disorders in children: Sociodemographic and clinical characteristics. Journal of the American Academy of Child and Adolescent Psychiatry, 31, 1070-1076. http://dx.doi.org/10.1097/00004583-199211000-00012

Lonigan, C. J., \& Vasey, M. W. (2009). Negative affectivity, effortful control, and attention to threatrelevant stimuli. Journal of Abnormal Child Psychology, 37, 387-399. http://dx.doi.org/10.1007/s10802-008-9284-y

Lyneham, H.J., \& Rapee, R.M. (2005). Agreement between telephone and in-person delivery of a structured interview for anxiety disorders in children. Journal of the American Academy of Child and Adolescent Psychiatry, 44, 274-282. http://dx.doi.org/10.1097/00004583-200503000-00012

Mansell,W., Clark, D. M., Ehlers, A., \& Chen, Y. P. (1999). Social anxiety and attention away from emotional faces. Cognition \& Emotion, 13, 673-690. http://dx.doi.org/10.1080/026999399379032

Mogg, K., \& Bradley, B. P. (1998). A cognitive-motivational analysis of anxiety. Behaviour Research and Therapy, 36, 809-848. http://dx.doi.org/10.1016/S0005-7967(98)00063-1 
Mogg, K. \& Bradley, B. P. (1999). Some methodological issues in assessing attentional biases for threatening faces in anxiety: a replication study using a modified version of the probe detection task. Behaviour Research and Therapy, 37, 595-604. http://dx.doi.org/10.1016/S0005-7967(98)00158-2

Mogg, K., \& Bradley, B. P. (2002). Selective orienting of attention to masked threat faces in social anxiety. Behaviour Research \& Therapy, 40, 1403-1414. http://dx.doi.org/10.1016/S00057967(02)00017-7

Mogg, K., Philippot, P., \& Bradley, B.P. (2004). Selective attention to angry faces in clinical social phobia. Journal of Abnormal Psychology, 113, 160-165. http://dx.doi.org/10.1037/0021843X.113.1.160

Monk, C. S., Nelson, E. E., McClure, E. B., Mogg, K., Bradley, B. P., Leibenluft, E., ... Pine, D. S. (2006). Ventrolateral prefrontal cortex activation and attentional bias in response to angry faces in adolescents with generalized anxiety disorder. The American Journal of Psychiatry, 163, 1091-1097. http://dx.doi.org/10.1176/appi.ajp.163.6.1091

Monk, C. S., Telzer, E. H., Mogg, K., Bradley, B. P., Mai, X., Louro, H. M., .. Pine, D. S. (2008). Amygdala and ventrolateral prefrontal cortex activation to masked angry faces in children and adolescents with generalized anxiety disorder. Archives of General Psychiatry, 65, 568-576. http://dx.doi.org/10.1001/archpsyc.65.5.568

Nauta, M. H., Scholing, A., Rapee, R., Abbott, M., Spence, S. H., \& Waters, A. M. (2004). Parent-report measure of children's anxiety: Psychometric properties and comparison with child-report in a clinical and normal sample. Behaviour Research and Therapy, 42, 413-439. http://dx.doi.org/10.1016/S00057967(03)00200-6

Perez-Edgar, K., Bar-Haim, Y., McDermott, J., Chronis-Tuscano, A., Pine, D. S., \& Fox, N. A. (2010). Attention bias to threat and behavioural inhibition in early childhood shape adolescent social withdrawal. Emotion, 10, 349-357. http://dx.doi.org/10.1037/a0018486

Pine, D. S., Mogg, K., Bradley, B, P., Montgomery, L., Monk, C. S., McClure, E., ... Kaufman, J. (2005). Attention bias to threat in maltreated children: Implications for vulnerability to stress-related psychopathology. American Journal of Psychiatry, 162, 291-296. http://dx.doi.org/10.1176/appi.ajp.162.2.291

Rapee, R. M., \& Heimberg, R. G. (1997). A cognitive-behavioral model of anxiety in social phobia. Behaviour Research and Therapy, 35, 741-756. http://dx.doi.org/10.1016/S0005-7967(97)00022-3

Rapee, R. M., Barrett, P. M., Dadds, M. R., \& Evans, L. (1994). Reliability of the DSM-III-R childhood anxiety disorders using structured interview: interrater and parent-child agreement. Journal of the American Academy of Child and Adolescent Psychiatry, 33, 984-992. http://dx.doi.org/10.1097/00004583-199409000-00008

Roy, A. K., Vasa, R. A., Bruck, M., Mogg, K., Bradley, B. P., Sweeney, M., .. Pine, D. S. (2008). Attention bias toward threat in paediatric anxiety disorders. Journal of the American Academy of Child and Adolescent Psychiatry, 47, 1189-1196. http://dx.doi.org/10.1097/CHI.0b013e3181825ace

Silverman, W. L., \& Albano, A. M. (1996). Anxiety Disorders Interview Schedule for DSM-IV: Child and Parent Versions. San Antonio, Psychological Corporation.

Silverman, W. K., Saavedra, L. M., \& Pina, A. A. (2001). Test-retest reliability of anxiety symptoms and diagnoses with the Anxiety disorders interview schedule for DSM-IV: Child and parent versions. Journal of the American Academy of Child and Adolescent Psychiatry, 40, 937-944. http://dx.doi.org/10.1097/00004583-200108000-00016

Spence, S. H. (1998). A measure of anxiety symptoms among children. Behaviour Research and Therapy, 36, 545-566. http://dx.doi.org/10.1016/S0005-7967(98)00034-5 
Spence, S. H., Donovan, C. and Brechman-Toussaint, M. (1999). Social skills, social outcomes, and cognitive features of childhood social phobia. Journal of Abnormal Psychology, 108, 211-221. http://dx.doi.org/10.1037/0021-843X.108.2.211

Stirling, L. J., Eley, T. C., \& Clark, D. M. (2006). Preliminary evidence for an association between social anxiety symptoms and avoidance of negative faces in school-age children. Journal of Clinical Child and Adolescent Psychology, 35, 440-445. http://dx.doi.org/10.1207/s15374424jccp3503 9

Taghavi, M. R., Dalgleish, T., Moradi, A. R., Neshat-Doost, H. T., \& Yule, W. (2003). Selective processing of negative emotional information in children and adolescence with generalised anxiety disorder. British Journal of Clinical Psychology, 42, 221-230. http://dx.doi.org/10.1348/01446650360703348

Telzer, E. H., Mogg, K., Bradley, B. P., Mai, X., Ernst, M., Pine, D. S. \& Monk, C. S. (2008). Relationship between trait anxiety, prefrontal cortex, and attention bias to angry faces in children and adolescents. Biological Psychology, 79, 216-222. http://dx.doi.org/10.1016/j.biopsycho.2008.05.004

Wadlinger, H. A., \& Isaacowitz, D. M. (2008). Looking happy: The experimental manipulation of a positive visual attention bias. Emotion, 8, 121-126. http://dx.doi.org/10.1037/1528-3542.8.1.121

Waters, A. M., Mogg, K., Bradley, B. P., \& Pine, D. S. (2008). Attentional bias for emotional faces in children with generalized anxiety disorder. Journal American Academy of Child and Adolescent Psychiatry, 47, 435-442. http://dx.doi.org/10.1097/CHI.0b013e3181642992

Waters, A. M., Neumann, D. L., Henry, J., Craske, M. G., \& Ornitz, E. M. (2008). Baseline and affective startle modulation by emotional faces in 4-8 year old high and low anxious children. Biological Psychology. 78, 10-19. http://dx.doi.org/10.1016/j.biopsycho.2007.12.005

Waters, A. M., Henry, J., Mogg, K., Bradley, B. P., \& Pine, D. S. (2010). Attentional bias towards angry faces in childhood anxiety disorders. Journal of Behavior Therapy and Experimental Psychiatry. 41, 158-164. http://dx.doi.org/10.1016/j.jbtep.2009.12.001

Waters, A. M., Kokkoris, L. K., Mogg, K., Bradley, B. P., \& Pine, D. S. (2010). The time course of attentional bias for angry faces in anxious children. Cognition \& Emotion. 24, 1173-1181. http://dx.doi.org/10.1080/02699930903274355

Watts, S. E., \& Weems, C. F. (2006). Associations among selective attention, memory bias, cognitive errors, and symptoms of anxiety in youth. Journal of Abnormal Child Psychology, 34, 841-852. http://dx.doi.org/10.1007/s10802-006-9066-3

Williams, J. M. G., Watts, F. N., Macleod, C., \& Mathews, A. (1997). Cognitive psychology and emotional disorders (2nd ed.). Chichester: Wiley.

Wittchen, H.-U., Stein, M. B., \& Kessler, R. C. (1999). Social fears and social phobia in a community sample of adolescents and young adults: prevalence, risk factors, and co-morbidity. Psychological Medicine, 29, 309-323. http://dx.doi.org/10.1017/S0033291798008174 